\title{
Las funciones de los trabajadores sociales sanitarios en atención primaria de salud: evolución, redefinición y desafíos del rol profesional
}

\author{
María C. Abreu Velázquez ${ }^{1}$; Vinita Mahtani Chugani²
}

Recibido: 28/11/2016 / Revisado: 23/01/2017 / Aceptado: 11/05/2017

Resumen. Los trabajadores sociales sanitarios fueron introducidos en la atención primaria de salud como resultado de la reforma sanitaria ocurrida en España, en los años 1980. Sus actuaciones estaban dirigidas inicialmente al abordaje de los problemas psicosociales individuales y a la intervención en la comunidad. Con el objetivo de analizar si el rol de estos profesionales ha ido evolucionado para adaptarse a las necesidades actuales de la institución y afrontar las nuevas demandas de la población, se llevó a cabo una investigación cualitativa, desde una perspectiva descriptiva, inductiva y evolutiva, mediante el examen de los programas y protocolos del Servicio Canario de Salud. Los resultados indican que se trata de un rol polifacético que se ha ido transformando para hacer frente a las nuevas necesidades de la población a la vez que se encaran desafíos que obligan a la redefinición del perfil profesional.

Palabras clave: investigación cualitativa; trabajo social sanitario; atención primaria; rol.

\section{[en] Functions of healthcare social workers in primary healthcare: evolution, redefinition and challenges of the professional role}

\begin{abstract}
Healthcare social workers were introduced to primary healthcare as a result of the healthcare reform that took place in Spain during the 1980s. Their activities were initially directed at addressing individual psychosocial problems and community intervention. Qualitative research was conducted from a descriptive, inductive and evolutionary perspective with the aim of analysing whether these professionals' role has evolved to adapt to the current needs of the institution and to face the new demands of the population, by way of an examination of the programmes and protocols of the Canary Islands Health Service (Servicio Canario de Salud). The results show that this is a multifaceted role, which has transformed in order to meet the new needs of the population at the same time as facing challenges that have forced a redefinition of the professional profile.
\end{abstract}

Key words: qualitative research; healthcare social work; primary care; role.

Sumario: Introducción. 1. Metodología. 2. Resultados. 2.1. Atención directa. 2.2. Apoyo social. 2.3. Promoción de la salud y participación comunitaria. 2.4. Investigación y docencia. 2.5. Otros roles y actitudes recomendados. 3. Discusión. 4. Conclusiones. 5. Referencias bibliográficas.

Cómo citar: Abreu Velázquez, M. C.; Mahtani Chugan, V. (2018) Las funciones de los trabajadores sociales sanitarios en Atención primaria de salud: evolución, redefinición y desafíos del rol profesional, en Cuad. trab. soc. 31(2), 355368.

\footnotetext{
Servicio Canario de Salud, España innavel@yahoo.es

2 Servicio Canario de Salud, España. vinitavivek@hotmail.com
} 


\section{Introducción}

Los trabajadores sociales entraron a formar parte de los equipos de atención primaria de salud a partir de la reforma sanitaria promulgada en la Ley 14/1986, de 25 de abril, General de Sanidad; hasta entonces habían estado circunscritos a los hospitales y dispositivos de salud mental. Este hecho se produjo como resultado del cambio de paradigma de atención a la salud en el que la dimensión social alcanzó una mayor relevancia, y por las propuestas formuladas en los años 1970 para la reforma del sistema sanitario. Según Hernández (2013), el acierto en incorporar a los trabajadores sociales a los equipos médicos creados en 1981 para dar una respuesta a la epidemia del síndrome del aceite tóxico, basada "en la interacción y la coordinación entre lo social y lo sanitario", favoreció su posterior inclusión en los nuevos equipos de Atención primaria.

En los años 1980, las actuaciones de los trabajadores sociales sanitarios de atención primaria se orientaron preferentemente hacia el abordaje de los problemas psicosociales individuales y familiares y a la intervención en la comunidad (Ituarte, 1990). Se veía al trabajador social como el profesional idóneo para emprender las actividades propias de la orientación comunitaria del nuevo modelo (Informe Insalud, 2002; Corrales-Nevado et al., 2012). En esta primera etapa, las funciones de estos profesionales no estaban reguladas y frecuentemente se solapaban y eran confundidas con los de los trabajadores sociales de los servicios sociales comunitarios (Ituarte, 1990). Esta circunstancia unida a la ausencia de sistemas de registro que proporcionaran una información detallada sobre sus actuaciones conformó una visión estereotipada del trabajo social sanitario (Colom, 2004) identificado frecuentemente con tareas burocráticas ligadas, principalmente, a la derivación a los recursos comunitarios (Corrales-Nevado et al., 2012).

El éxito y la expansión del nuevo modelo de atención primaria no conllevó el desarrollo, en la misma medida, del trabajo social sanitario. Entre las posibles causas se ha señalado la dificultad para contemplar la dimensión social de la salud porque esta "encajaría mal en un modelo de servicios sanitarios absolutamente dominados por el paradigma médico-biológico" (Fernández, 2008) o porque no se vían como parte integrante del modelo biopsicosocial de atención a la salud (Gijón y Colom,
2016). Por otro lado, los trabajadores sociales se han encontrado en una posición débil dentro de las instituciones sanitarias debido al lugar que ocupan frente a otras disciplinas que gozan de un estatus científico del que carece el Trabajo Social en España. Ello ha facilitado la penetración de otras profesiones en los espacios tradicionales del Trabajo Social. Para Burgos (2003), el desconocimiento de la administración sanitaria y de gran parte de los profesionales sanitarios de sus cometidos supone una amenaza para el Trabajo Social y sus consecuencias son: la sobrecarga asistencial, la discriminación con respecto a otros profesionales de la salud y una escasa dotación de trabajadores sociales en los distintos servicios sanitarios.

Este escenario profesional se enmarca en un contexto global donde se están generando importantes retos para los sistemas sanitarios como son el envejecimiento poblacional y el aumento de las enfermedades crónicas (Van Lerberghe, 2008) a lo que se suma el crecimiento de las desigualdades en salud. Para hacer frente a estos desafíos se vienen proponiendo cambios organizativos de gran calado en los que se pretende colocar el centro de gravedad en la atención primaria en salud, por su capacidad para proporcionar cuidados de carácter integral y su continuidad a lo largo del ciclo vital de las personas, actuando como gestora y coordinadora de casos. Algunos de los cambios planteados se apoyan en la redefinición y creación de nuevos perfiles profesionales (Isaacson, Summers-Holtrop, Cohen, Ferrer y McKee, 2012; Corrales-Nevado et al., 2012) con el objetivo, entre otros, de potenciar la coordinación de los equipos de atención primaria con los proveedores de servicios sociales, sociosanitarios y otros, que operan en la comunidad, creando redes de apoyo para la provisión de cuidados. Estas ideas se introducen en España donde se gesta un "movimiento pro gestión de caso" (Casado, 2012) como una de las estrategias que pretenden mejorar la atención a la cronicidad y la dependencia.

Los trabajadores sociales sanitarios en el primer nivel asistencial resultan claves para el abordaje de problemas de salud complejos donde se asocian patologías crónicas y necesidades sociales (Reckrey et al., 2014; Craig et $a l ., 2016)$ y por sus técnicas para enlazar a los pacientes con los servicios sociales y sociosanitarios, proporcionados por otros sistemas de protección, superando la fragmentación de 
los servicios (Barber, Coulourides-Kogan, Riffenburgh y Enguidanos, 2015) para garantizar el continuum asistencial y el abordaje de la cronicidad.

El aumento de la prevalencia de enfermedades asociadas a necesidades sociales contribuye al incremento de los costes de la asistencia sanitaria, añadiendo un mayor grado de complejidad, a la par que se registran resultados en salud más reducidos en población con problemas psicosociales. Los trabajadores sociales, en un contexto de trabajo multidisciplinar, abordan estos factores psicosociales aumentando la adherencia terapéutica y contribuyendo a la mejoría global del paciente (Claiborne y Vandenburgh, 2001).También ayudan a los pacientes a identificar sus problemas y a fijar un plan de actuación mejorando sus habilidades y la propia gestión de la salud (Enguidanos, Coulourides, Keefe, Geron y Katz,, 2011).Teniendo en cuenta que cerca del 80 por ciento de los pacientes que frecuentan los centros de atención primaria presentan ansiedad y depresión (Craig et al., 2016), los estudios sugieren que los trabajadores sociales tienen un papel clave en la disminución de los factores sociales asociados a estas patologías (idem). En la última década los resultados de las investigaciones sugieren que los beneficios del trabajo social, en un amplio rango de problemas y poblaciones, pueden ser cuantificables (Mullen y Shuluk, 2012; McGregor et al., 2016).

Todo ello está dirigiendo a los trabajadores sociales sanitarios hacia la redefinición de sus roles para desarrollar nuevas habilidades (Geissler-Piltz, 2011) y para enfrentar el desafío de ser sustituidos, en algunas de sus funciones, por otros profesionales sanitarios, mayormente enfermeros (Pineda y Sánchez-Robles, 2006; Auerbach y Manson, 2007; Reisch, 2012; Cleak y Turczynski, 2014).

En Canarias, la realidad de los trabajadores sociales sanitarios en atención primaria no es ajena a los problemas y desafíos ya señalados. Las funciones dentro de los programas y protocolos del Servicio Canario de Salud dirigidos al abordaje de las enfermedades están suficientemente reconocidas; pero, teniendo en cuanta la necesidad de intensificar las actuaciones dirigidas al abordaje de la cronicidad y la dependencia, se hace necesario examinar el rol normativo atribuido a los trabajadores sociales sanitarios y constatar si se ha ido adaptando a los cambios propuestos para la atención primaria en salud. Habitualmente, no se investiga cómo se produce la evolución de los roles profesionales en las instituciones a lo largo del tiempo ni si los cambios de orientación son efectivos para los fines propuestos (Isaacson et al., 2012). Por ello, el objetivo del presente trabajo es analizar las funciones atribuidas a los trabajadores sociales sanitarios de los centros de atención primaria, en los programas y protocolos de abordaje de las enfermedades y constatar si se adecúan a las necesidades actuales de la institución para afrontar las nuevas demandas de la población.

\section{Metodología}

Se diseñó un estudio cualitativo, inicialmente de tipo descriptivo inductivo, mediante un análisis de documentos con una perspectiva evolutiva. Durante este proceso se identificó un marco de análisis deductivo que se combinó con el análisis inductivo inicial. Se recogieron todos los programas, protocolos y documentos relacionados con la atención primaria, publicados por el Servicio Canario de Salud durante el periodo 1988-2008. Se consultaron los archivos bibliográficos y las bases de datos informatizadas de la institución. No se hizo selección de muestra sino que se incluyeron todos los documentos publicados en el periodo de estudio. En el Cuadro 1 se describen las características de los documentos analizados. 
Cuadro 1: Relación de documentos analizados

\begin{tabular}{|c|c|c|c|c|c|c|c|}
\hline Documento & Año & Tipo & Formato & $\begin{array}{l}\text { Extensión } \\
\text { Páginas }\end{array}$ & $\begin{array}{l}\text { Consta } \\
\text { Autores }\end{array}$ & $\begin{array}{c}\text { Participa } \\
\text { Trabajador } \\
\text { social }\end{array}$ & $\begin{array}{c}\text { Participa } \\
\text { Trabajador } \\
\text { social } \\
\text { Revisión }\end{array}$ \\
\hline \multicolumn{8}{|c|}{ Instituto Nacional de la Salud } \\
\hline $\begin{array}{l}\text { Funciones Equipos de } \\
\text { Atención Primaria }\end{array}$ & No consta & Manual & Papel & 33 & No & No consta & No consta \\
\hline $\begin{array}{l}\text { Organización Centros } \\
\text { de Salud }\end{array}$ & No consta & Manual & Papel & 61 & No & No consta & No consta \\
\hline $\begin{array}{l}\text { Enfermedad. Cardio- } \\
\text { vascular }\end{array}$ & 1993 & Programa & Papel & No consta & No & No & No consta \\
\hline \multicolumn{8}{|c|}{ Servicio Canario de Salud } \\
\hline $\begin{array}{l}\text { Malos tratos Mujeres y } \\
\text { Menores }\end{array}$ & 1999 & Protocolo & Papel & 101 & $\mathrm{Si}$ & $\mathrm{Si}$ & $\mathrm{Si}$ \\
\hline $\begin{array}{l}\text { Enfermos Pre y Post } \\
\text { trasplantados }\end{array}$ & 1999 & Protocolo & Papel & 51 & $\mathrm{Si}$ & $\mathrm{Si}$ & $\mathrm{Si}$ \\
\hline $\begin{array}{l}\text { Enfermedad Cardiovas- } \\
\text { cular }\end{array}$ & 2000 & Programa & Electrónico & 92 & $\mathrm{Si}$ & No & No \\
\hline Salud Bucodental & 2000 & Programa & Electrónico & 52 & $\mathrm{Si}$ & No & $\mathrm{Si}$ \\
\hline Personas Mayores & 2002 & Programa & Electrónico & 303 & $\mathrm{Si}$ & $\mathrm{Si}$ & $\mathrm{Si}$ \\
\hline $\begin{array}{l}\text { Enfermedad Cardiovas- } \\
\text { cular }\end{array}$ & 2003 & Programa & Electrónico & 54 & $\mathrm{Si}$ & No & No \\
\hline $\begin{array}{l}\text { Alzheimer/ Otras De- } \\
\text { mencias }\end{array}$ & 2003 & $\begin{array}{l}\text { Guía } \\
\text { Práctica } \\
\text { Clínica }\end{array}$ & Electrónico & 86 & $\mathrm{Si}$ & $\mathrm{Si}$ & $\mathrm{Si}$ \\
\hline Violencia de Género & 2003 & Protocolo & Electrónico & 91 & $\mathrm{Si}$ & $\mathrm{Si}$ & $\mathrm{Si}$ \\
\hline Personas Cuidadoras & 2003 & Manual & Electrónico & 153 & $\mathrm{Si}$ & No & $\mathrm{Si}$ \\
\hline Salud Infantil & 2005 & Programa & Electrónico & 265 & $\mathrm{Si}$ & $\mathrm{Si}$ & $\mathrm{Si}$ \\
\hline Salud Afectivo Sexual & 2005 & Programa & Electrónico & 98 & $\mathrm{Si}$ & $\mathrm{Si}$ & $\mathrm{Si}$ \\
\hline Aulas de Salud & 2006 & Manual & Electrónico & 12 & & & \\
\hline $\begin{array}{l}\text { Salud Mental Infanto/ } \\
\text { Juvenil }\end{array}$ & 2006 & Programa & Electrónico & 75 & $\mathrm{Si}$ & No & No \\
\hline Salud Infantil & 2007 & Programa & Electrónico & 265 & $\mathrm{Si}$ & $\mathrm{Si}$ & $\mathrm{Si}$ \\
\hline Climaterio & 2007 & Programa & Electrónico & & $\mathrm{Si}$ & No & No \\
\hline $\begin{array}{l}\text { Salud Mental en A. } \\
\text { Primaria }\end{array}$ & 2008 & Manual & Electrónico & 168 & $\mathrm{Si}$ & No & No \\
\hline $\begin{array}{l}\text { Continuidad de Cui- } \\
\text { dados }\end{array}$ & 2008 & Protocolo & Electrónico & 42 & $\mathrm{Si}$ & $\mathrm{Si}$ & No \\
\hline
\end{tabular}


Al tratarse de un estudio de carácter evolutivo y documental no fue necesario efectuar consideraciones éticas específicas. Ambas autoras participaron en el diseño del estudio, en la elaboración de los resultados y en la presentación de los mismos; el trabajo de campo fue llevado a cabo por la primera autora.

Una vez recopilados los documentos, se procedió a la lectura general; posteriormente se extrajo información del contexto de elaboración de cada uno y de todos los apartados en los que se hacía referencia a la disciplina de trabajo social y su rol.

Seguidamente, se procedió a la lectura, codificación y el análisis de los manuales. Primero se generó un listado de códigos y, a partir de ellos, se elaboraron las categorías de análisis que se refinaron mediante un proceso de comparación y concordancia entre investigadoras. En este proceso se identificó, como documento core, el Documento Marco: Rol profesional de Trabajo Social en la atención primaria de salud, reconocido normativamente en la Instrucción $n^{\circ}$ 3/06 de la Directora del Servicio Canario de Salud, el cual se utilizó como marco teórico para definir las categorías de análisis. Por ello, finalmente se optó por el análisis de la información mediante la aplicación de un marco teórico, framework analysis (Ritchie y Spencer, 1994).
Los documentos examinados pertenecen a dos periodos distintos de la sanidad pública canaria. El primero corresponde a los primeros años de la reforma, en el marco del antiguo Instituto Nacional de la Salud, y el segundo, a partir de 1993, cuando se produce la transferencia de las competencias del Estado central a la Comunidad Autónoma de Canarias y se crea el Servicio Canario de Salud. Hubo dificultad para encontrar los documentos de la primera etapa en formato papel por lo que se recurrió a los fondos bibliográficos de la propia institución.

\section{Resultados}

Las funciones se agruparon en cinco áreasatención directa, apoyo social, promoción de la salud y participación comunitaria e investigación y docencia- correspondientes a las descritas en el Documento Marco: Rol profesional de Trabajo Social en la atención primaria de salud y en la Instrucción $n^{\circ} 3 / 06$.

Otros aspectos reflejados en los documentos fueron también analizados.

En el Cuadro 2 se exponen el desarrollo del rol normativo, según el área de intervención, enfermedad, población y año en el que se registró la función.

Cuadro 2: Desarrollo del rol según área de intervención, enfermedad, población y año.

\begin{tabular}{|c|c|c|c|c|c|}
\hline Área/Sector de población & Año & $\begin{array}{l}\text { Atención } \\
\text { Directa }\end{array}$ & $\begin{array}{l}\text { Apoyo } \\
\text { Social }\end{array}$ & $\begin{array}{l}\text { Promoción } \\
\text { Prevención }\end{array}$ & $\begin{array}{c}\text { Investigación } \\
\text { Docencia }\end{array}$ \\
\hline $\begin{array}{l}\text { Funciones Equipos de atención } \\
\text { primaria }\end{array}$ & $1986 / 88$ & + & & +++ & +++++ \\
\hline EnfermedadCardiovascular & $1993 / 2003$ & +++ & + & +++ & \\
\hline Malos tratos a mujeres/niños & 1999 & + & & + & \\
\hline Enfermos Pre y pos trasplantados & 1999 & +++ & & & \\
\hline Salud Bucodental & 2000 & & & & \\
\hline Personas Mayores & 2002 & ++++ & + & ++ & \\
\hline Alzheimer y otras demencias & 2003 & ++++ & & & \\
\hline Violencia deGénero & 2003 & ++++ & & & \\
\hline Personas cuidadoras & 2003 & ++ & & & \\
\hline Salud Infantil & $2005 / 2007$ & +++++ & ++ & +++ & \\
\hline Salud afectivosexual & 2005 & & & ++ & \\
\hline Aulas de Salud & 2006 & & & ++ & \\
\hline Salud MentalInfanto Juvenil & 2006 & & & + & \\
\hline Climaterio & 2007 & + & & & \\
\hline
\end{tabular}




$\begin{array}{lllll}\text { Salud Mentalen atención primaria } & 2008 & + & \\ \begin{array}{l}\text { Continuidad de Cuidados para } \\ \text { atención domiciliaria }\end{array} & 2008 & +++++ & ++ & +\end{array}$

Leyendas: +1 función ++2 funciones +++3 funciones ++++4 funciones +++++5 o más funciones

\subsection{Atención directa}

Hace referencia a las intervenciones directas que se realizan con los usuarios y pacientes a nivel individual y familiar. Se trata de la dimensión que aglutina el mayor número de actividades. Es la categoría de análisis donde se han recabado más datos.

En la primera etapa de la reforma, el trabajador social asume el rol de asesor y se le reconoce como el profesional conocedor de los recursos sociales:

- Informar, asesorar u orientar al individuo y a la familia sobre los recursos existentes y su correcta utilización (Manual de normas y funciones de los equipos de atención primaria/1986/88).

- Atención en consulta a demanda de la población priorizando familias en riesgo (ídem)

- Realizar gestiones, informes sociales y visitas a organismos. (Ídem).

- Informar sobre derechos y deberes; sobre servicios del centro de salud; también sobre recursos sociales (Idem).

Hacia 1999, en los enunciados de las funciones, se plasman una mayor precisión. Por ejemplo, describiendo la metodología:

- La trabajadora social debe realizar la valoración psicosocial/la intervención /derivación y el seguimiento de los pacientes al alta (Protocolo de intervención psicosocial en enfermos pre y pos trasplantados/1999).

En los años siguientes se van haciendo más precisas:

- El trabajador social es el gestor de casos con problemática social (Programa de atención a las personas mayores/2002).
- Debe prestar atención psicosocial a personas mayores con demencia (Ídem).

- Debe intervenir en los casos de malos tratos (Idem).

Es más específica en la atención a las demencias:

- Dar información completa y veraz para atender las necesidades psicosociales de los pacientes y sus familiares (Guía de Práctica Clínica Atención al Alzheimer y Otras Demencias/2003).

- Debe participar en la atención multidisciplinar a pacientes con enfermedad de Alzheimer (Idem).

Y en la atención a menores:

- Debe identificar factores de riesgo social en la infancia (Salud Infantil, 2005).

- Debe estar alerta ante indicadores de maltrato (Idem)

Se describe de manera pormenorizada la atención a la población infantil con discapacidad:

- Plan de intervención social de la trabajadora social como gestora de casos (Idem).

- Falta de información sobre recursos, dificultades, etc. (Idem)

- Dificultades con escolaridad, ocupación, integración, etc. (Idem)

- Problemas laborales vinculados al cuidado

- Dificultades económicas derivadas de la situación de discapacidad

- Sentimiento o situación de aislamiento

- Sobrecarga de los cuidadores

- Sobre implicación continuada

El programa de Salud Mental Infanto-Juvenil desarrolla funciones para los trabajadores sociales sanitarios de Atención especializada; 
mientras que las acciones preventivas a nivel comunitario se consideran una parcela de los trabajadores sociales sanitarios de Atención primaria.

Las acciones de prevención de la enfermedad y promoción de la salud mental se desarrollarán por los sectores sanitario, educativo y social, en los que se verán involucrados:

- Los Equipos de Atención Primaria: Pediatras, médicos de familia, personal de enfermería y trabajadores sociales (Salud Mental Infanto-Juvenil, 2006).
Para la población adulta, la actividad en salud mental se equipara a la de actores que operan fuera del sistema sanitario:

Otras medidas (en el abordaje de la distimia) que pueden resultar de utilidad son:

- solicitar la colaboración del trabajador social

- grupos de autoayuda

- actividades sociales de ocio y socialización (Salud Mental en Atención Primaria, 2008)

En el marco específico de la atención familiar se formula una variedad de funciones que se exponen en la Tabla 1.

Tabla 1: Funciones en la atención a la familia

\begin{tabular}{lll}
\hline \multicolumn{1}{c}{ FUNCIÓN } & \multicolumn{1}{c}{ AÑO } & PROGRAMA \\
Informar, orientar y asesorar sobre recursos & 1986 & $1,2,4,6$ \\
Captación y seguimiento de familias de riesgo & 1986 & 1,6 \\
Tratar/intervenir en la problemática familiar & 2000 & $1,3,5$ \\
Trabajar el empoderamiento & 2003 & 1 \\
Canalizar hacia recursos & 2003 & 4 \\
Favorecer el respiro familiar & 2003 & 4 \\
Valoración sociofamiliar ante factores de ries- & 2005 & 1 \\
go social en niños & & \\
Establecer un plan de intervención ante facto- & 2005 & 1 \\
res de riesgo social familiar en niños & & \\
Abordaje dificultades existentes y/o potencia- & 2005 & 1 \\
les en el entorno familiar de los niños & & \\
Organización de los cuidados & 2005 & 1 \\
Situación laboral/ocupacional & 2005 & 1 \\
Atender la sobrecarga & 2005 & 1 \\
Explorar el entorno familiar & 2005 & 1 \\
Valorar la situación económica & 2005 & 1 \\
Dar información ajustada a las necesidades & 2005 & 1,2 \\
Apoyar el proceso de toma decisiones & 2005 & 1 \\
Apoyo en la elaboración afrontamiento & 2005 & 1 \\
Apoyar el proceso de adaptación & 2005 & 1 \\
\hline
\end{tabular}

Leyendas: 1 Programa de Salud Infantil 2. Alzheimer y Otras demencias. 3. Continuidad de Cuidados 4. Taller Cuidadoras. 5. Cardiovascular 6. Manual de normas y funciones de equipos de atención primaria. 
En el área de atención directa también se encuadran las funciones correspondientes a la actividad de coordinación con los recursos sociales y sociosanitarios que operan en el territorio. El trabajador social se considera como el profesional de "enlace" en la coordinación con las instituciones, las asociaciones y los líderes comunitarios. Su papel es liderar la coordinación con los servicios sociales para la atención directa a los usuarios y para realizar un seguimiento junto a otros organismos, entre los cuales se señalan: "la Fiscalía, los colegios, Dirección General del Menor y los servicios especializados" (Salud Infantil, 2005). Con las personas dependientes la coordinación se desarrollará mediante protocolos. Para ello, debe conocer los recursos institucionales y asociativos y confeccionar una base de datos de los recursos sociosanitarios.

En el Protocolo de actuación ante la violencia de género en el ámbito doméstico, no se estipulan las competencias de cada profesión; la "valoración social" forma parte de una "valoración biopsicosocial" general.

Con respecto al trámite o la gestión de prestaciones sociales se ha encontrado una única referencia: "debe gestionar la calificación de minusvalía" (Salud Infantil, 2005)

En esta dimensión de análisis se hace referencia al ámbito de actuación del trabajador social sanitario: "en consulta y en domicilio" (Manual de normas y funciones de los equipos de atención primaria 1986-88).

Desde la primera etapa de la reforma se alude al modo en que debe producirse el acceso de los usuarios a los servicios del trabajador social sanitario: "a demanda de los usuarios" "atención en programas" "por derivación de otros profesionales" (Idem).

Igualmente, se encontraron referencias a la obligación de registrar los datos recabados en el curso de las actuaciones, incluyendo el diagnóstico social: "registrar sus actividades" (idem); "incorporar a la historia el diagnostico social" (Prevención y control enfermedad cardiovascular, 2000; Protocolo del Servicio de Continuidad de Cuidados para la atención domiciliaria, 2008)

Otras actividades reflejadas en los documentos consideran la dimensión deontológica:

- debe solicitar el consentimiento informado (Atención a enfermos pre y post trasplantados, 1999).

- mantener el secreto profesional (Actuación ante la violencia de género en atención primaria, 2003)

En lo que respecta el ejercicio de sus actividades profesionales, los programas y protocolos señalan que deben sustentarse dentro de un método: "valorar....", "realizar el estudio...", "tratar problemáticas", "intervención social". Estableciendo "un plan de cuidados junto al médico y el enfermero o plan de intervención" así como: "elaborar programas junto a los miembros del equipo de atención primaria (Manual de normas y funciones de los equipos de atención primaria 1986-88); "evaluar programas" (Prevención y control enfermedad cardiovascular, 2000); "plantear objetivos y nuevas actividades" (ídem) .

En el Cuadro 3 se relacionan las funciones en la atención directa a usuarios según el programa o protocolo correspondiente. 
Cuadro 3. Desarrollo de las funciones en la atención directa a usuarios según programa o protocolo.

\begin{tabular}{|c|c|c|c|c|}
\hline Programa / Protocolo & $\begin{array}{l}\text { Valoración } \\
\text { Social }\end{array}$ & $\begin{array}{l}\text { Diagnostico } \\
\text { Social }\end{array}$ & Intervención & $\begin{array}{l}\text { Coordinación } \\
\text { con recursos }\end{array}$ \\
\hline $\begin{array}{l}\text { Funciones Equipos de } \\
\text { atención primaria }\end{array}$ & & & + & + \\
\hline $\begin{array}{l}\text { Enfermedad Cardiovas- } \\
\text { cular }\end{array}$ & + & + & + & + \\
\hline $\begin{array}{l}\text { Malos tratos mujeres/ } \\
\text { menores }\end{array}$ & & & + & + \\
\hline $\begin{array}{l}\text { Enfermos pre y post } \\
\text { trasplantados }\end{array}$ & + & & + & + \\
\hline $\begin{array}{l}\text { Personas mayores en } \\
\text { atención primaria }\end{array}$ & + & & + & gestor de casos \\
\hline $\begin{array}{l}\text { Alzheimer y otras de- } \\
\text { mencias }\end{array}$ & + & & + & + \\
\hline Violencia de Género & & & + & \\
\hline Personas cuidadoras & + & & + & + \\
\hline Salud infantil & $\begin{array}{l}+ \\
\text { *atención } \\
\text { específica a } \\
\text { niños con dis- } \\
\text { capacidad }\end{array}$ & + & & gestor de casos \\
\hline Climaterio & + & & + & \\
\hline $\begin{array}{l}\text { Salud Mental en Aten- } \\
\text { ción Primaria }\end{array}$ & & & $\begin{array}{l}+ \\
\text { abordaje de } \\
\text { la distimia }\end{array}$ & \\
\hline $\begin{array}{l}\text { Continuidad de cuida- } \\
\text { dos para la atención } \\
\text { domiciliaria }\end{array}$ & + & + & + & gestor de casos \\
\hline
\end{tabular}

\subsection{Apoyo social}

Bajo este epígrafe se incluyen funciones relacionadas con el impulso de las redes sociales de apoyo como recurso en salud mediante la utilización de los grupos de ayuda mutua y el fomento del voluntariado como estrategia.

- Se deben promover redes de apoyo tanto formal como informal, sobretodo para las personas dependientes. (Protocolo del Servicio de Continuidad de Cuidados para la atención domiciliaria, 2008)

- El conocimiento de los recursos asociativos y sus actividades también se asocia a esta área (Manual de normas y funciones de los equipos de atención primaria 1986-88.)

- El profesional debe participar activamente en la génesis de actividades grupales, apo- yar la generación de grupos, promoción del voluntariado y la creación de grupo de ayuda. (Manual de normas y funciones de los equipos de atención primaria 1986-88; Protocolo del Servicio de Continuidad de Cuidados para la atención domiciliaria, 2008).

\subsection{Promoción de la salud y participación comunitaria.}

Bajo este enunciado se agrupan aquellas funciones que recogen estrategias para la participación de la comunidad, la promoción de la salud y el autocuidado. Para ello, el trabajador social debe coordinarse con otras instituciones, asociaciones y líderes de la comunidad con el fin de impulsar acciones para la promoción de la salud mediante la educación para la salud. Es responsable de estudiar la socioestructura de la comunidad, sus condiciones socioeconó- 
micas y culturales, de identificar la dinámica social y detectar situaciones carenciales y los factores de riesgo. Para ello debe conocer los recursos asociativos y/o conocer todas las organizaciones. Deberá participar en la generación de grupos de pacientes, apoyar a los grupos ya existentes y las actividades de promoción de la salud.

- En el caso de la prevención y promoción en salud mental se pide que está función esté dirigida a la población infantil y adolescente. Salud Mental Infanto- Juvenil/2006

Como actividades específicas se encuentran:

- Participar en la proyección comunitaria de los programas.

- Uso de la propaganda (carteles y folletos) y de los medios de comunicación para realizar esta difusión (Programa de atención a la salud afectiva sexual y reproductiva. PASAR, 2005).

- Contribuir a la celebración de los días internacionales y otros eventos organizados ya sea por la propia institución sanitaria o por otros colectivos y asociaciones ( Prevención y control enfermedad cardiovascular, 2000).

Su participación se equipara a la de otros profesionales sanitarios de atención primaria: El trabajador social tiene el rol de profesional de la salud junto a médicos y enfermeros (Aulas de Salud, 2006).

\subsection{Investigación y docencia}

En este apartado se clasifican las actividades de investigación propia, la difusión de los resultados y la participación en estudios.

Se han encontrado tres referencias en un único documento:

- Se realizaran investigaciones encaminadas a la adaptación y desarrollo de los métodos y técnicas de trabajo social dentro de la Zona de Salud (Manual de normas y funciones de los equipos de atención primaria 1986/88).

- Estudiar las condiciones socio- económicas y culturales de la zona y difundir los resultados de la investigación al resto del Equipo (Idem).

- Participar en los proyectos de investigación que el Equipo de Atención Primaria desarrolle según las necesidades y recursos de la zona (Idem).

\subsection{Otros roles y actitudes recomendados}

Se encontraron apelaciones a los trabajadores sociales sanitarios para practicar determinadas habilidades dentro de la propia organización; así se les conmina a: trabajar, comunicarse y realizar interconsultas con otros miembros del equipo sanitario. En el mismo sentido, están obligados a: derivar al médico de familia las patologías detectadas en su propia consulta; informar sobre los recursos sociales y sobre el grado de satisfacción de los usuarios. Se les encomienda proporcionar formación al equipo sobre el trabajo social y colaborar en la formación de profesionales sanitarios de pre y posgrado.

De igual forma, se le asigna un rol de enlace entre los niveles asistenciales de atención primaria y especializada, enunciándose como una obligación la función de coordinarse con otros servicios y departamentos del sistema sanitario y a establecer vías o protocolos de coordinación con los trabajadores sociales de otros niveles asistenciales. Con respecto a los pacientes, debe apoyar su asistencia a los servicios especializados.

Se le pide una implicación activa y ética:

- Debe implicarse personalmente en adquirir hábitos de vida saludable ( Prevención y control enfermedad cardiovascular, 2000)

- Debe ejercer una implicación activa en la detección precoz de la violencia de género y la intervención (Actuación ante la violencia de género en atención primaria, 2003).

Es la puerta de entrada, para los usuarios, al sistema sanitario, junto al acceso a médicos y enfermeros: "el médico, el enfermero y el trabajador social del primer nivel de salud representan para la mayoría de los pacientes la puerta de entrada al sistema sanitario y, por tanto, el primer contacto profesional "(Guía de Práctica Clínica de la enfermedad de Alzheimer y otras demencias, 2003).

Se destaca un área donde debe ejercer un liderazgo: 
- El trabajador social debe liderar la coordinación (Protocolo del Servicio de Continuidad de Cuidados para la atención domiciliaria, 2008).

- El trabajador social es responsable de la coordinación con otros servicios (sociales, educativos).

\section{Discusión}

En el análisis de rol normativo denota un perfil polifacético de los trabajadores sociales sanitarios en cuyo marco desarrollan una gran variedad de intervenciones, abarcando tanto los problemas psicosociales individuales, mediante el Trabajo Social de caso, la gestión de casos y otros, así como la promoción de la salud y la intervención comunitaria. Este perfil coincide con el que se ha descrito en estudios realizados en otros países y en distintos modelos sanitarios occidentales (Raghallaigh et al., 2013; McGregor et al., 2016). Sin embargo, este perfil podría no ser extrapolable a la totalidad del Sistema Nacional de Salud, dado que solo se ha examinado un único servicio de salud y por la ausencia de estudios similares, tratándose de una limitación de este trabajo.

La atención directa a usuarios es el principal escenario de las intervenciones lo que sugiere una orientación individualista en el abordaje de los problemas sociales. Los menores, especialmente aquellos que presentan discapacidad, y las personas mayores son los sectores de población donde se hace un mayor énfasis; en estos colectivos a los trabajadores sociales sanitarios se les reconoce como los gestores de casos, cuando está asociada una problemática social. Este foco puesto en las personas mayores, impulsado por los cambios demográficos, conecta con la tendencia del trabajo social a nivel internacional (Mirabito, 2012).

La mirada del Trabajo Social sanitario a la familia ha ido evolucionando, inicialmente concebida como un sistema de interacciones, atendiendo a un enfoque ecosistémico, al sumar una concepción más utilitarista -la familia como proveedora de cuidados- adaptada a las nuevas exigencias del sistema sanitario.

Las enfermedades cardiovasculares y las demencias son las afecciones donde se ha hecho un mayor hincapié, indicando la prioridad de las patologías crónicas. Cuando a estas se suman necesidades sociales complejas se apun- ta al trabajador social sanitario como el gestor de los casos en la continuidad de cuidados. En este sentido se le identifica significativamente como el conocedor y el asesor en recursos y servicios sociales dirigidos a los pacientes $\mathrm{y}$ a sus familias. El asesoramiento familiar es clave en un modelo de cuidados característico como el nuestro, el del sur de Europa, basado en un alto nivel de cuidado informal y un escaso desarrollo de los servicios de atención residencial.

Los resultados parecen destacar el papel de los trabajadores sociales sanitarios en la coordinación con los recursos comunitarios y como el profesional que enlaza al equipo de atención primaria con la comunidad y, dentro del propio sistema sanitario, con el nivel de atención especializada para garantizar el continuum asistencial.

Los planes de atención individualizados, el uso de los servicios adecuados a cada fase de la enfermedad y el cuidado interdisciplinar son esenciales para que las personas puedan permanecer en la comunidad (Reckrey et al., 2014), incluso en las etapas más avanzadas. El método de gestión de casos, que guarda muchas similitudes con la propia disciplina del Trabajo Social (Kanter, 2011; Rimbau, 2012), se presenta como el más adecuado, considerándose implícitamente que los trabajadores sociales tienen la formación pertinente para desempeñar este rol (Sarabia, 2009).

Los resultados de este trabajo parecen revelar la escasa relevancia del diagnóstico social, en el rol normativo, lo que podría guardar relación con una disposición reactiva ante las problemáticas psicosociales que se abordan luego en la práctica cotidiana.

La participación de los trabajadores sociales sanitarios en la elaboración y revisión de un programa o protocolo puede determinar el grado de implicación en la atención a un problema de salud concreto. En salud mental no participa ni en la creación ni en la revisión de los protocolos, mientras otros profesionales sanitarios les atribuyen el rol de colaborador en la atención directa a pacientes, y limitado a la distimia.

El presente estudio sugiere que su aportación en salud mental estaría siendo subestimada ya que otras investigaciones señalan que el Trabajo Social, en el abordaje de la depresión y la ansiedad asociada, puede ayudar a las personas a mejorar sus mecanismos de afrontamiento y también puede ofrecer atencio- 
nes tempranas y preventivas, actuando sobre los determinantes sociales de la salud. Otros estudios (Leroy, 2004; Fraser et al., 2004; Hawkins, 2009; Craig et al., 2016) subrayan la efectividad de las intervenciones en la auto regulación de las emociones, en la mejora de calidad de las relaciones interpersonales etc.

El protocolo de atención a la violencia de género, donde la valoración social no está atribuida a los trabajadores sociales sanitarios de una manera precisa, puede ser un ejemplo de cómo no siempre la participación en la elaboración de un protocolo se convierte en una oportunidad para delimitar el espacio profesional en un contexto multidisciplinar. Otros programas, como el de la salud bucodental, sin participación ni actividad alguna, ponen de manifiesto algunos de los espacios hacia donde es necesario extender el radio de acción.

Cuando los trabajadores sociales sanitarios participan en la configuración de los programas y protocolos, el rol normativo es más coincidente con el académico. En este trabajo se encontró que los profesionales habían participado de una manera significativa en la configuración del rol normativo; las referencias a tareas burocráticas o al trámite de recursos fueron irrelevantes. Dado que en la literatura se suele aludir a la burocratización de las actividades (Burgos, 2003; Corrales-Nevado et al., 2012) y al malestar ante la imagen estereotipada de tramitador de recursos que se proyecta sobre los trabajadores sociales (Colom, 2004), lo que puede estar produciéndose es un falta de concordancia entre el rol normativo y el rol operativo, esto es lo que realmente se hace en la práctica. Al respecto, García y Sobremonte (2002) advierten de que: "los trabajadores sociales sanitarios son profesionales que actúan a demanda de los usuarios y de otros profesionales, en función de las propias necesidades y urgencias de estos, contribuyendo a la construcción de una imagen estereotipada". Ello podría producirse como resultado de un sentimiento de inferioridad que conduce a los trabajadores sociales a la "auto depreciación" (Sticher-Gil, 1993) y por una "actitud camaleónica" que les lleva a adaptarse a aquellos roles y patrones profesionales que le son asignados (Terbuyken, 1997). Por otro lado, numerosos estudios subrayan que los médicos y los enfermeros no comprenden bien los distintos roles de los trabajadores sociales (Keefe, Geron y Enguidanos, 2009) siendo identificados mayormente con el trámite y gestión de ayu- das y recursos (Sánchez Reyes, 2016; Gijón y Colom, 2016), lo que contribuye a la construcción de la imagen distorsionada de los trabajadores sociales sanitarios y a un obstáculo para el trabajo en equipo.

En la actualidad se están produciendo cambios en el sistema sanitario implantándose nuevos perfiles para algunas profesiones biomédicas, con funciones de los trabajadores sociales, contando con un potente apoyo institucional. Por el contrario, los trabajadores sociales sanitarios carecen del respaldo indispensable para desempeñar el rol emanado de las normas de la propia organización sanitaria.

Estos cambios también están relacionados con la transformación de las bases filosóficas sobre las que se asentó la reforma sanitaria que, en sus inicios, puso el foco en el estudio de las condiciones socioeconómicas, culturales y en el conocimiento de los recursos institucionales de la zona, respondiendo a la orientación comunitaria con la que nació el nuevo modelo de atención primaria y la necesidad del estudiar el entorno de las personas y su medio social para un abordaje integral de la salud. Siguiendo esta línea, algunos programas pusieron el acento en señalarles, a los trabajadores sociales sanitarios, la obligación de implicarse en la consecución personal de hábitos de vida saludable y de contar con habilidades como el liderazgo, la responsabilidad y el secreto profesional.

Paulatinamente, este enfoque ha ido diluyéndose (Informe Insalud, 2002) para centrarse, de manera casi hegemónica, en la atención individual y la gestión de casos. En consecuencia, el rol de los trabajadores sociales sanitarios ha ido evolucionando para la adaptarse a la atención a la cronicidad y a las personas mayores.

Finalmente, en la investigación es el área profesional donde se ha observado un menor desarrollo del rol profesional con una presencia prácticamente residual que se remonta a los inicios de la reforma. La clave estaría en aumentar los estudios con usuarios para demostrar la efectividad del Trabajo Social sanitario en la mejora de los resultados en salud, sobre todo en poblaciones con necesidades sociales diagnosticadas, y para determinar su contribución a la disminución de los costes en la prestación de servicios sanitarios. Es fundamental incrementar la investigación aplicada para avalar la presencia de trabajadores sociales en los equipos sanitarios asistenciales, ya que la ausencia de un cuerpo de evidencia robusto 
sobre la efectividad de los servicios ofrecidos por los trabajadores sociales sanitarios ha disuadido a los gestores sanitarios de apostar por el incremento de profesionales (Keefe et al., 2009).

Según McGregor et al. (2016) las intervenciones de los trabajadores sociales producen mejoras significativas en el funcionamiento de los pacientes y en su propia gestión de la salud, reducen la morbilidad psicosocial, incrementan la percepción subjetiva en salud y disminuyen las barreras en el acceso al tratamiento y al mantenimiento de la salud.

\section{Conclusiones}

El estudio de las funciones de los trabajadores sociales de atención primaria apunta significativamente hacia un rol polifacético con múltiples componentes; no se han encontrado evidencias de que su misión sea realizar tareas burocráticas.
Estas características deberían tenerse en cuenta en el diseño de las estrategias de abordaje a la cronicidad que se están llevando a cabo en el sistema sanitario público.

El rol normativo ha ido evolucionando para dirigirse hacia la atención a la cronicidad. Se debe seguir profundizando en estos cambios, de acuerdo a las tendencias del Trabajo Social contemporáneo, hacia enfoques más clínicos y a la realización de estudios sobre la efectividad de las intervenciones. Sería igualmente indispensable llevar a cabo más investigaciones sobre el rol profesional en el conjunto del sistema sanitario en España y sobre aspectos concretos de la práctica, tales y como: el papel en la coordinación sociosanitaria y de enlace entre los distintos niveles asistenciales.

El objetivo debería ser trabajar para una mayor coherencia entre el rol académico, el normativo y el operativo, trascendiendo la separación entre la teoría y la práctica, y describiendo los procedimientos y los procesos de la práctica asistencial.

\section{Referencias bibliográficas}

Auerbach, C., Manson, S.E. y Heft La Porte, H. (2007). Evidence that supports the value of social work in hospitals. Social Work in Health Care, 44(4), 17-32.

Barber R., Coulourides-Kogan, A., Riffenburgh, A. y Enguidanos, S. (2015). A role for social workers in improving care settings transitions: a case study. Social work in health care. 54(3), 177-192. DOI 10.108000981389 .2015 .100527

Burgos Varo, M.L. (2003). Evolución del trabajo social en la atención a la salud de nuestro país durante el último siglo. Trabajo Social y Salud, 46, 85-99.

Casado, D. (2012). Aplicaciones y demanda de la gestión de casos en España. Servicios Sociales y Política Social, 105, 23-32.

Claiborne, N. y Vanderburgh, H. (2001). Social workers' role in disease management. Health \& Social Work, 26, 217-225.

Cleak, H.M. y Turczynski, M. (2014). Hospital social work in Australia: emerging trends o more of the same? Social work in health care 53(3), 199-213 Doi 10.1080/00981389.2013.873516

Colom, D. (2004). Estudios de la calidad percibida en los servicios de trabajo social de salud: un cambio para la mejora. Trabajo Social y Salud, 49, 39-60.

Corrales-Nevado, D. et al. (2012). Continuidad de cuidados, innovación y redefinición de papeles profesionales en la atención a pacientes crónicos y terminales. Informe SESPAS 2012. Gaceta Sanitaria, 63-68. DOI:10.1016/j.gaceta.2011.09.032

Craig, S., Frankford, R., Allan, K., Charmaine, W. et al. (2016). Self-reported patient psychosocial needs in integrated primary health care: A role for social work in interdisciplinary teams. Social work in health care, 55, 41-60. DOI: 1080/00981389.2015.1085483

Enguidanos, S., Coulourides K.A., Keefe, B., Geron, S.M. y Katz, L. (2011).Patient-centered approach to building problem solving skills among older primary care patients: problems identified and resolved. Journal of Gerontological Social Work, 53(3), 276-291.

Fernández-Díaz J. (2008) Complementariedad de la intervención social en el proceso salud. Agathos. 3, 4-8.

García Humayor, A. y Sobremonte de Mendicuti, E. (2002). Mejorando la calidad: el desarrollo de procesos de intervención social en las organizaciones sanitarias. Trabajo Social y Salud, 42, 29-50. 
Geissler-Piltz, B. (2011). How social workers experience supervision: results of an empirical study in the healthcare sector. Journal of Social Intervention: Theory and Practice. 20, 1-5

Gijón Sánchez, M.T. y Colom, D. (2016). Debates transdisciplinares en torno a la especialización del trabajo social sanitario. Respuestas transdisciplinares en una sociedad global: Aportaciones desde el Trabajo Social. Logroño: Universidad de La Rioja.

Hernández-Martin, G. (2013). El trabajo social en la construcción de lo sanitario (1970-1986). Agathos, 4, 26-32

INSALUD. (2012). Informe: Atención Primaria en el INSALUD: 17 años de experiencia. Recuperado de: http://www.ingesa.msssi.gob.es/estadEstudios/documPublica/pdf/Completo.pdf

Isaacson, N., Summers-Holtrop, J., Cohen, D., Ferrer, R.L. y McKee, M.D. (2012). Examining role change in primary care practice. Journal of primary care community health, 3, 195-200. DOI $10.1177 / 2150131911428338$

Ituarte, A. (1990) Trabajo social en salud. Historia, situación actual, perspectivas. Trabajo social y Salud, 6, 51-2.

Kanter, J. (2011) Clinical case management. En: Jerold R. Brandell (ed.), Theory \& practice in clinical social work (pp.561- 586). Thousand Oaks, California: SAGE.

Keefe, B., Geron, S.M. y Enguidanos, S. (2009) Integrating social workers into primary care: physician and nurse perception of roles, benefits and challenges. Social work in health care, 48(6), 579-596 DOI: 10. 1080/00981380902765592

Mc Gregor, J., Mercer, S.W. y Harris, F.M.(2016). Health benefits of primary care social work for adults with complex health and social needs: a systematic review. Health Social Care Community. Doi 10.1111/ hsc/12337[Epub ahead of print]

Mullen, E.J. y Shuluk, J. (2011). Outcomes of social work intervention in the context of evidence-based practice. Journal of Social Work, 11(1), 49-6.

Raghallaigh, M.N., Allen, M., Cunniffe, R. y Quin, S. (2013). Experiences of social workers in primary care in Ireland. Social work in health care, 52(10), 930-946. Doi 10.1080./00981389.2013.834030.

Reckrey, J.M. et al. (2014). The critical role of social workers in home-based primary care. Social work in health care, 53, 330-343.

Reisch, M. (2012). The challenges of health care reform for hospital social work in the United States. Social Work in Health Care, 51, 873-893.

Rimbau-Andreu C. (2012) La gestión de caso, conceptualización, contexto y presencia social actual. Servicios Sociales y Política Social, 105, 33-44.

Ritchie, J. y Spencer, L. (1994). Qualitative data analysis for applied policy research. En: A. Bryman y R.G. Burgess (eds.), Analyzing qualitative data (pp.173-194).

Sarabia, A. (2009). Los agentes de la gestión de caso. Perfil profesional. Revista Políticas Sociales en Europa, Monográfico: Gestión de caso (y métodos afines) en servicios sanitarios y sociales, 25-26. 193-204.

Sánchez Reyes, M. (2016). Las necesidades sociales originadas a consecuencia de la enfermedad en pacientes adultos hospitalizados. (Tesis doctoral. Universidad Complutense de Madrid, Facultad de Trabajo Social, ES).

Sticher-Gil, B. (1993). Übergänge von der Sozialpsychiatrie zur Sozialarbeit. Studium Praxis, . 70, 3-7.

Pineda del Pino, A.I. y Sánchez Robles, M. (2006) Análisis del presunto intrusismo profesional en el ámbito del trabajo social sanitario: la enfermera de enlace. Trabajo Social y Salud, 55, 173-186.

Terbuyken, G. (1997) Verstehen und Begleiten. Konzeptionelle Überlegungen zum Selstverständnis von Sozialarbeiter/-innen in der Psychiatrie. Soziale Arbeit, 2, 38-48.

Van Lerberghe, W. (2008). The world health report 2008: primary health care: now more than ever. Ginebra: World Health Organization. 